\title{
Slippage effect on energy modulation in seeded free-electron lasers with frequency chirped seed laser pulses
}

\author{
Chao Feng, ${ }^{1,2}$ Haixiao Deng, ${ }^{1}$ Guanglei Wang, ${ }^{1,2}$ Dong Wang, ${ }^{1}$ and Zhentang Zhao,** \\ ${ }^{1}$ Shanghai Institute of Applied Physics, Chinese Academy of Sciences, Shanghai 201800, China \\ ${ }^{2}$ University of Chinese Academy of Sciences, Beijing 100049, China \\ Dao Xiang
}

SLAC National Accelerator Laboratory, Menlo Park, California 94025, USA

(Received 8 February 2013; published 27 June 2013)

\begin{abstract}
Free-electron lasers (FELs) seeded with external lasers hold great promise for generating high power radiation with nearly transform-limited bandwidth in the soft $\mathrm{x}$-ray region. However, it has been pointed out that the initial seed laser phase error will be amplified by the frequency up-conversion process, which may degrade the quality of the output radiation produced by a harmonic generation scheme. In this paper, theoretical and simulation studies on frequency chirp amplification in seeded FEL schemes with slippage effect taken into account are presented. It is found that the seed laser imperfection experienced by the electron beam can be significantly smoothed by the slippage effect in the modulator when the slippage length is comparable to the seed laser pulse length. This smoothing effect allows one to preserve the excellent temporal coherence of seeded FELs in the presence of large frequency chirp in the seed laser. Our studies show that the tolerance on frequency chirp in the seed laser for generating nearly transformlimited soft x-ray pulses in seeded FELs is much looser than previously thought and fully coherent radiation at nanometer wavelength may be reached with current technologies.
\end{abstract}

PACS numbers: $41.60 . \mathrm{Cr}$

\section{INTRODUCTION}

The recent success of self-amplified spontaneous emission (SASE) based $x$-ray free-electron laser (FEL) facilities $[1,2]$ has paved the way for novel types of experiments in many scientific disciplines. While the radiation from a SASE FEL has excellent transverse coherence, it typically has rather limited temporal coherence. There are many techniques (e.g. x-ray resonant inelastic scattering, spectroscopic studies of correlated electron materials, etc.) that could benefit from improved temporal coherence. To meet these scientific needs, various FEL seeding schemes such as coherent harmonic generation (CHG) [3], high-gain harmonic generation (HGHG) [4], and echo-enabled harmonic generation (EEHG) $[5,6]$ have been proposed and experimentally demonstrated [7-13].

In seeded harmonic generation FELs, typically an external coherent seed laser pulse is first used to interact with electrons in a short undulator, called modulator, to produce energy modulation in the electron beam. This energy modulation is then converted into density modulation by a small chicane, called the dispersion section (DS). Taking

\footnotetext{
*Corresponding author. zhaozt@sinap.ac.cn
}

Published by the American Physical Society under the terms of the Creative Commons Attribution 3.0 License. Further distribution of this work must maintain attribution to the author(s) and the published article's title, journal citation, and DOI. advantage of the fact that the density modulation shows Fourier components at $n$th high harmonics of the seed, intense radiation at shorter wavelengths can be generated. Ideally, it is anticipated that the output radiation in a seeded FEL should inherit the properties of the seed laser with its bandwidth close to the Fourier transform limit. However, there are several challenges in implementing seeding schemes at extremely high harmonics. For example, too large initial energy spread will constrain the possibility of working at high harmonic number for a single stage; the jitter of the central beam energy will result in a large fluctuation of the shot-to-shot output pulse energy; the nonuniform distributions of the local parameters will affect the longitudinal profile of the radiation pulse, which may degrade the coherence of the output radiation. In particular, the initial insignificant errors compared to the seed wavelength may be amplified by the harmonic up-conversion process and will become large relative to a much shorter wavelength. For example, the electron beam shot noise will be amplified by $n^{2}$, which may overwhelm the external seeding source [14]. More recently, attentions have been turned to errors from the imperfection of the seed laser. It has been pointed out that if there is a frequency chirp in the seed pulse, the chirp in the electron microbunching turns out to be roughly multiplied by the harmonic number $n$ $[15,16]$. As a result, generation of nearly transform-limited radiation at $1 \mathrm{~nm}$ wavelength from a commercial $800 \mathrm{~nm}$ Ti:sapphire seed laser requires that the extra timebandwidth product contributed by the seed phase chirp 
should be no more than one in a million of the ideal seed pulse [15], which is well beyond the state-of-the-art laser technology.

The conclusions in Refs. $[15,16]$ were drawn under the assumption that the phase of the energy modulation directly copies the phase of the seed laser after the modulator. The seed power and frequency variations as a function of time due to the slippage effect in the modulator were neglected. This assumption is reasonable in the case of using an ideal seed laser with infinite pulse length and flat spectral phase distribution. However, for a realistic seed laser pulse with finite duration, the slippage effect on the energy modulation should be considered, especially for the case when the laser pulse width is comparable to the slippage length in the modulator.

As the continuous progress in laser technologies, such as the chirped-pulse amplification (CPA) [17], have made ultrashort and high intensity laser pulses available, many seeded FEL facilities now adopt short seed laser pulses. For instance, the seed pulse duration of the FERMI seeded FEL is around 150 fs (FWHM) [18], and the Shanghai deep ultraviolet FEL uses an ultrashort seed pulse of about 80 fs (FWHM) [19]. However, the temporal and spatial quality of the laser beam will be degraded due to the propagation process, nonlinear effects, or inhomogeneous doping concentration in the amplifying media of the CPA system [15]. FELs seeded with ultrashort laser pulses have been intensively studied in Refs. [20,21] with a focus on wavelength tunability. Recently, the impact of the linear frequency chirp on the output radiation of a seeded FEL has been experimentally studied for the first time in Ref. [22]. In this paper, a new model that considers the slippage effect in the modulator is developed to describe the energy modulation with a frequency chirped seed laser pulse. It is found that the frequency chirp induced by the seed laser can be smoothed by the slippage effect, when the slippage length in the modulator is comparable to the pulse length of the seed laser. For ultrashort UV seed lasers with FWHM pulse length around $16 \mathrm{fs}$, this condition is generally met with a modulator with $\sim 30$ undulator periods. For longer seed laser pulses with FWHM pulse length around $80 \mathrm{fs}$, we propose using a modulator tuned at the subharmonic of the UV seed laser to boost the slippage length to a similar level as the laser width. Three-dimensional simulations have been carried out for a soft x-ray facility based on seeded FEL schemes to illustrate how the sensitivity of the FEL bandwidth to the initial frequency chirp can be significantly reduced by a proper design of the modulator. Our studies show that the tolerance on laser frequency chirp for generating nearly transform-limited soft x-ray pulses in seeded FELs is much looser than that suggested in $[15,16]$ and fully coherent radiation at nanometer wavelength may be reached with current technologies.

\section{ENERGY MODULATION WITH SLIPPAGE EFFECT}

Here we consider a planar undulator with a sinusoidal magnetic field in the vertical direction and a period length $\lambda_{u}$. In the laboratory frame, the undulator magnetic field of the modulator is

$$
\vec{B}_{y}=B_{0} \sin \left(k_{u} z\right) \vec{y}
$$

where $B_{0}$ is the undulator peak magnetic field and $k_{u}=$ $2 \pi / \lambda_{u}$ is the wave number of the undulator. The orbit of a relativistic electron in such a field is approximately a sine wave, and the velocity of the electron is given by

$$
\vec{v}(t)=v_{z}(t) \vec{z}-\frac{K c}{\gamma} \cos \left(\omega_{u} t\right) \vec{x},
$$

where $v_{z}$ is the electron velocity in the $z$ direction, the undulator parameter is $K \approx 0.934 B_{0} \lambda_{u}$ with $B_{0}$ in Tesla and $\lambda_{u}$ in centimeter, $c$ is the speed of light, $\omega_{u}$ is the angular frequency of the orbit, and $\gamma$ is the relativistic electron energy. The electron's transverse velocity induced by the undulator magnet is

$$
v_{x}(t)=-\frac{K c}{\gamma} \cos \left(\omega_{u} t\right)
$$

Since the average velocity of the electron is approximately constant, $v=c \sqrt{1-1 / \gamma^{2}}, v_{z}$ can be calculated by

$$
\begin{aligned}
v_{z}(t) & =c \sqrt{1-1 / \gamma^{2}-K^{2} \cos ^{2}\left(\omega_{u} t\right) / \gamma^{2}} \\
& \approx c-\frac{1+K^{2} / 2}{2 \gamma^{2}} c-\frac{K^{2} c}{4 \gamma^{2}} \cos \left(2 \omega_{u} t\right) .
\end{aligned}
$$

So the average electron longitudinal velocity is

$$
\bar{v}_{z} \approx c\left(1-\frac{1+K^{2} / 2}{2 \gamma^{2}}\right) \text {. }
$$

Assuming a seed laser pulse with a Gaussian power distribution of rms width $\sigma_{s}$, central wavelength at $\lambda_{s}$, and a linear frequency chirp $\alpha$ is used here. The electric field distribution along the electron beam can be represented as

$$
E(s)=E_{0} e^{-s^{2} / 4 \sigma_{s}^{2}} e^{i\left(k_{s} s+\alpha s^{2}+\phi_{0}\right)},
$$

where $s$ is the position along the electron bunch, $E_{0}$ is the peak electric field of the seed laser, $k_{s}=2 \pi / \lambda_{s}$ is the wave number of the seed laser, and $\phi_{0}$ is the initial carrier envelope phase of the laser. According to the resonant condition, the radiation overtakes the electron beam by one radiation wavelength per undulator period, which is called the slippage effect. Considering the slippage effect, the electric field and frequency distribution of the seed laser will vary with time, and Eq. (6) should be rewritten as

$$
E(s, t)=E_{0} e^{-[s-p(t)]^{2} / 4 \sigma_{s}^{2}} e^{i\left\{k_{s}[s-p(t)]+\alpha[s-p(t)]^{2}+\phi_{0}\right\}},
$$


where $p(t)=\left(c-\overline{\boldsymbol{v}}_{z}\right) t$ is the relative position of the seed pulse with respect to the electron beam. In a planar undulator, the electron has transverse wiggling motion and the longitudinal "figure-eight" oscillation. Such a trajectory gives rise to energy exchange between the electron and the laser electric field. The energy change of the electron can be calculated by

$$
\gamma_{m}(s, t)=\frac{e}{m c^{2}} E(s, t) \cdot v_{x}(t),
$$

where $e$ and $m$ are the charge and mass of the electron. Integrating Eq. (8) with respect to $t$, we arrive at an expression for the energy modulation along the electron bunch after passing through the modulator:

$$
\gamma_{m}(s)=\int_{0}^{L_{\mathrm{mod}} / c} \frac{e}{m c^{2}} E(s, t) \cdot v_{x}(t) d t
$$

where $L_{\text {mod }}$ is the length of the modulator.

When using an ultrashort seed pulse with the pulse length $\sigma_{s}$ comparable with the slippage length $N \lambda_{s}$ in the modulator, where $N$ is the period number of the modulator, part of the electron beam will be slipped over by the whole seed pulse and interacts with each cycle of the seed laser. So the energy modulation amplitude and the phase of the energy modulation will be averaged by the whole seed laser instead of directly copying the seed pulse. Figures 1 and 2 give the energy modulation properties when using an ultrashort seed laser with FWHM pulse length of $3 \lambda_{s}$ and a relatively large frequency chirp $\alpha=0.16 / \lambda_{s}{ }^{2}$. The period number of the modulator is $N=20$. For comparison of different models, we plug Eqs. (6) and (7) into Eq. (9) to calculate energy modulation with and without slippage effect, respectively. The results are illustrated in Fig. 1. The energy modulation amplitude is significantly reduced when considering the slippage effect, which means that the peak power of the seed laser and the strength of the DS

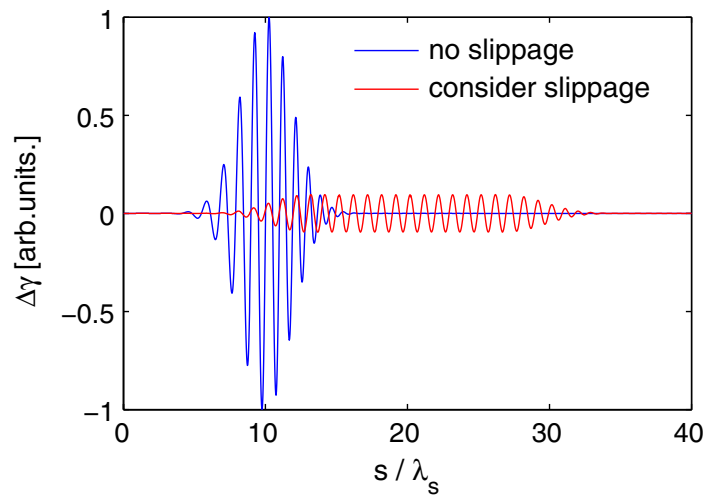

FIG. 1. Energy modulation with an ultrashort seed laser pulse. The energy modulation amplitude is uniform in the central part when considering the slippage effect using Eq. (7) (red line). For comparison, the energy modulation that directly copies the electric field is shown with a blue line.

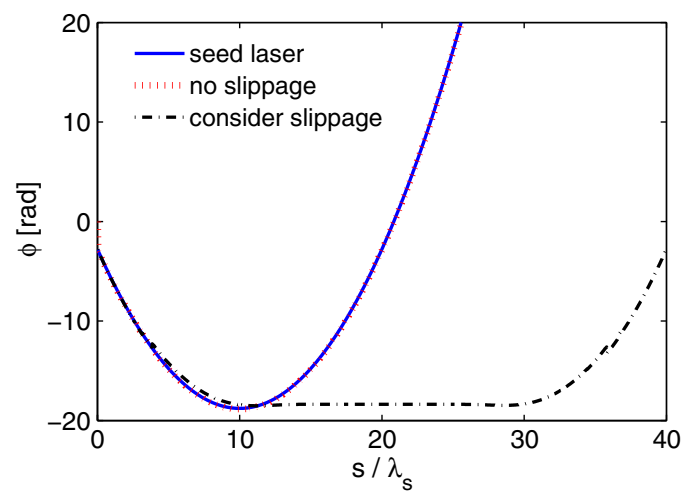

FIG. 2. Spectral phase distributions of the seed laser and energy modulations: quadratic phase in the seed laser (blue line) results in quadratic phase in the energy modulation when the slippage effect is neglected using Eq. (6) (red dots); when slippage effect is considered using Eq. (7) (black dash-dotted line), the phase distribution of the energy modulation is nearly flat in the central part.

should be properly enhanced to obtain sufficient spatial bunching in the electron beam $[20,21]$. It is also found that the slippage effect creates a uniform region (UR) in the energy modulation, where the energy modulation amplitude and phase distributions are nearly flat. Figure 2 gives the phase distributions of the seed laser and energy modulations along the electron bunch. When the slippage effect is neglected, the energy modulation has a quadratic phase that directly copies the spectral phase of the linear chirped in the seed laser. With the slippage effect taken into account, the spectral phase distribution is nearly flat in the central part.

To fully characterize the energy modulation in both the time and frequency domains and compare it with the seed laser pulse, we use the time-frequency distribution functions, which are appropriate tools to interpret the instantaneous carrier frequency, because they can simultaneously describe the temporal and spectral behavior of a signal by revealing time-resolved spectral structure. The Wigner distribution (WD) function [23,24] has the simplest form among the usually used time-frequency distribution functions and has a good marginal property:

$$
W(s, \omega)=\int \gamma(s-x / 2) \gamma^{*}(s+x / 2) e^{-i \omega x} d x,
$$

where * denotes the complex conjugate and $\omega$ is the carrier frequency of the energy modulation. WD intuitively shows the frequency distribution of the laser pulse or the energy modulation along the longitudinal direction, which makes it possible to analyze the frequency chirp quantitatively. Figure 3 shows the plots of WDs of the seed laser and energy modulations for different $N$. The vertical axis of Fig. 3 stands for the carrier frequency of the seed laser or the energy modulation. The carrier frequency is normalized by the central frequency of the seed laser. Figure 3(a) 

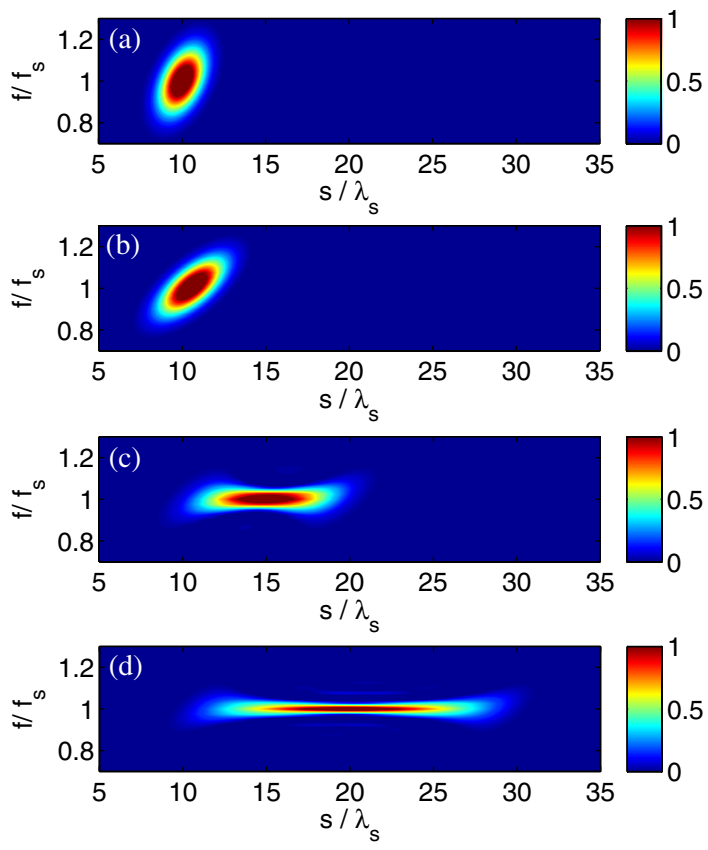

FIG. 3. Wigner distributions of the seed laser with FWHM pulse length of $3 \lambda_{s}$ (a) and energy modulations (b)-(d) for different period numbers of the modulator $(N=1,10,20)$. The frequency chirp in the seed laser pulse is $\alpha=0.16 / \lambda_{s}{ }^{2}$.

gives the WD of the seed laser pulse, where a considerable linear frequency chirp is clearly seen. When $N=1$, a linear chirp appears in the energy modulation as shown in Fig. 3(b). This chirp is significantly reduced in the central part when $N=10$ [Fig. 3(c)]. Meanwhile, the duration of the energy modulation with constant frequency begins to increase as the modulator length increases [Fig. 3(d)], which creates a UR with quite flat spectral phase.

From Figs. 3(c) and 3(d), one can find that with the slippage length longer than or comparable to the laser pulse width, the initial seed laser frequency chirp only leads to chirp in the lateral parts of the energy modulation. Note, with the modulation amplitudes in the lateral parts much smaller than that of the central part, the bunching at high harmonics would be negligible at these lateral regions. Therefore, the frequency chirp in the lateral parts will not significantly affect the final radiation bandwidth.

\section{HGHG AND EEHG WITH SHORT SEED PULSES}

For the HGHG scheme, after passage through the modulator section, the electron beam is sent into a DS to convert the energy modulation into density modulation. The density modulation of the electron beam can be measured by the bunching factor [4], which has a maximum value of unity. The properties of the coherent radiation at the very beginning of the radiator are mainly determined by the electron density distribution.
Consider an ideal beam with constant energy and current, assuming an initial Gaussian beam energy distribution with an average energy of $\gamma_{0}$ and rms energy spread $\sigma_{\gamma}$, the initial longitudinal phase space distribution can be written as

$$
f_{0}(p)=\frac{N_{0}}{\sqrt{2 \pi}} e^{-\left(p^{2} / 2\right)}
$$

where $N_{0}$ is the number of electrons per unit length and $p=\left(\gamma-\gamma_{0}\right) / \sigma_{\gamma}$ is the energy deviation of a particle normalized to the rms energy spread. After interacting with the seed laser pulse, the electron energy is changed to $p+A(s)$, where $A(s)=\gamma_{m}(s) / \sigma_{\gamma}$ is the energy modulation amplitude, and the distribution function becomes

$$
f_{1}(p, s)=\frac{N_{0}}{\sqrt{2 \pi}} \exp \left\{-[p-A(s)]^{2} / 2\right\} .
$$

Sending then the electron beam through the DS with normalized strength $B=k_{s} R_{56} \sigma_{\gamma} / \gamma_{0}$ converts the longitudinal coordinate to $s+B p / k_{s}$, where $R_{56}$ is the dispersive strength, and makes the final distribution function

$$
f_{\mathrm{HGHG}}(p, s)=\frac{N_{0}}{\sqrt{2 \pi}} \exp \left\{-\left[p-A\left(k_{s} s-B p\right)\right]^{2} / 2\right\} .
$$

Integration of the formula over $p$ gives the beam density distribution along the electron beam $N_{d}(s)=$ $\int_{-\infty}^{\infty} d p f_{\mathrm{HGHG}}(p, s)$. Then the $n$th local bunching factor at position $s$ can be written as

$$
\begin{aligned}
b(s, n)= & \frac{1}{\sqrt{2 \pi}} \int_{s-l / 2}^{s+l / 2} d x \int_{-\infty}^{\infty} \exp \left(-i n k_{s} x\right) \\
& \times \exp \left\{-\left[p-A\left(k_{s} x-B p\right)\right]^{2} / 2\right\} d p / l,
\end{aligned}
$$

where $l$ is the length of the chosen part of the electron beam.

The optimized strength of DS for $n$th harmonic bunching is $R_{56} \approx\left(1+0.81 n^{-2 / 3}\right) / A_{m} k_{s}$, where $A_{m}$ is the maximal value of the energy modulation amplitude. A large bunching factor at high harmonics relies on the formation of sharp density spikes, which requires large energy modulation amplitude. For an HGHG FEL, the energy modulation amplitude $A$ should be $n$ times larger than the initial energy spread to give a considerable value of bunching factor at $n$th harmonic [25,26]. However, as shown in Fig. 1, the energy modulation reduces for the lateral parts, which leads to broadened density spikes that cut off higher harmonics. This results in increasingly short pulses as the harmonic number increases. Here we adopted an ultrashort seed laser with FWHM pulse length of $17 \lambda_{s}$ and a frequency chirp of $\alpha=0.006 / \lambda_{s}{ }^{2} . N$ is chosen to be 30 to make the slippage length in the modulator comparable to the pulse length of the seed. We assume that the maximal energy modulation amplitude induced by the seed laser is 10 times larger than the initial energy spread. The local 
bunching factor distributions for different harmonics are calculated by Eq. (14), where the length of each slice is equal to the seed wavelength, $l=\lambda_{s}$. The calculation results are illustrated in Fig. 4. The bunching pulse length gets shorter at higher harmonics, which will change the harmonic spectral phase, as described in Ref. [16]. While the bunching at the fundamental wavelength roughly follows the energy modulation amplitude and phase, the bunching at high harmonics only samples phase from the UR of the energy modulation. Figure 5 shows the WDs of the seed laser, bunching factors at fundamental and 10th harmonic of the seed. The fundamental bunching inherits the frequency distribution of the energy modulation and has frequency chirps at lateral parts of the bunching pulse, where the bunching factor is much smaller than the central part. For the 10th harmonic, sufficient bunching only appears in the region with relatively large energy modulation amplitude, where the phase distribution is quite flat. So this slippage effect prevents the initial seed laser frequency chirp from broadening the final bandwidth of the high harmonic radiation.

Generally, the coherence property of the radiation could be quantified by the time-bandwidth product (TBP) factor, which can be simply defined as

$$
T=\Delta k \Delta \tau,
$$

where $\Delta k$ is the spectral bandwidth and $\Delta \tau$ is the pulse duration. $T$ has minimal value $T_{L}$ for a transform-limited pulse and will grow as the phase error increases. Figure 6 shows TBPs of the bunching factors as a function of the modulator period number for different harmonics of the seed. The bunching pulse distribution and the phase of the bunching are calculated by Eq. (14). $T$ is FWHM of the bunching pulse and has been normalized by $T_{L}$ in Fig. 6. As one can find from Fig. 6, the normalized TBP tends to decrease as the period number increases. The normalized TBPs for fundamental and 6th harmonic bunching are close to one (transform-limited pulse) when

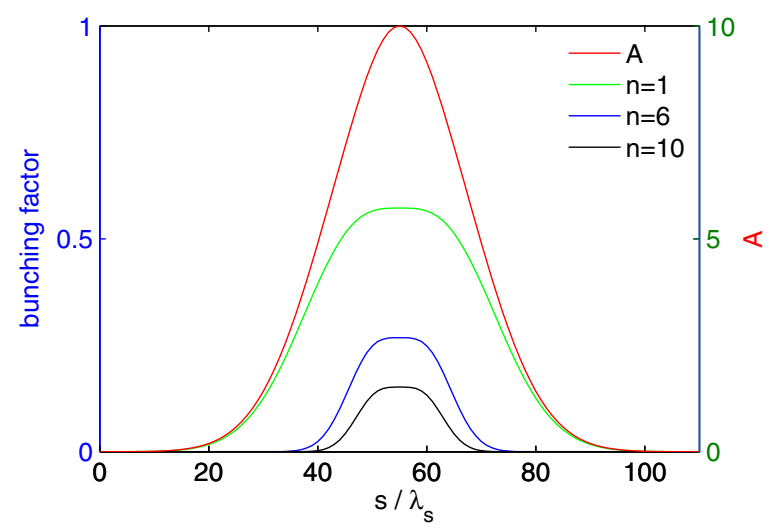

FIG. 4. Energy modulation amplitude and corresponding local bunching factor distributions along the electron beam for different harmonics of the seed $(n=1,6$, and 10).
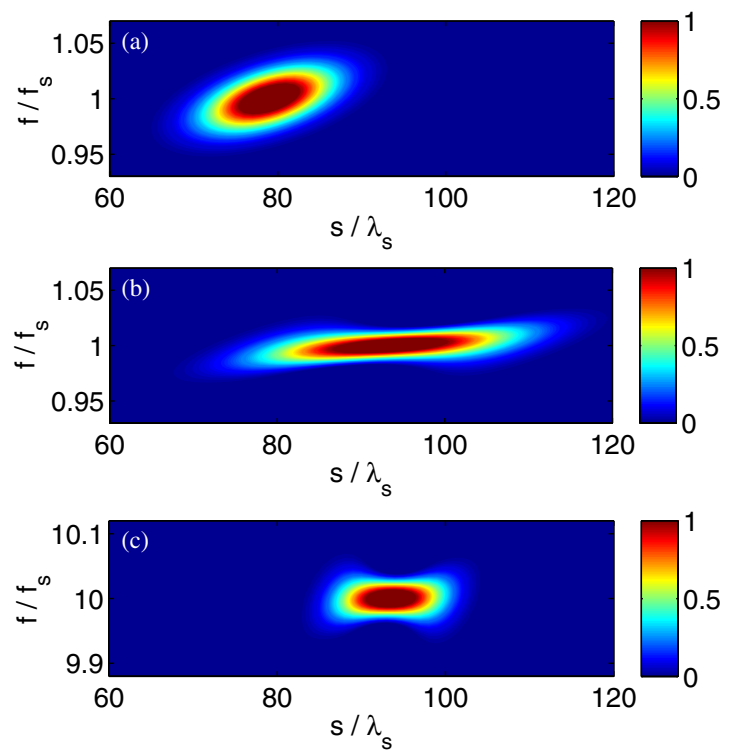

FIG. 5. Wigner distributions of the seed laser with FWHM pulse length of $17 \lambda_{s}$ (a) and local bunching factor at fundamental (b) and 10th harmonic (c) of the seed. The frequency chirp in the seed laser pulse is $\alpha=0.006 / \lambda_{s}{ }^{2}$.

$N$ is larger than 40. However, for 10th harmonic, the normalized TBP has a minimal value close to one when $N=30$. After that, the TBP starts to grow due to the nonlinear effect of the frequency chirp in the seed laser. The phase distributions of the bunching in the UR of energy modulation for various harmonics are shown in Fig. 7. The period number is chosen to be 30 [Fig. 7(a)] and 50 [Fig. 7(b)] to perform a comparison. For $N=30$, the phase distributions are quite flat in the UR of energy modulation for fundamental and high harmonics, which will result in transform-limited pulses. For a larger period number, $N=50$, a small frequency chirp that is reverse to the chirp in the seed laser appears in the central part. This reversed frequency chirp is insignificant at low harmonics,

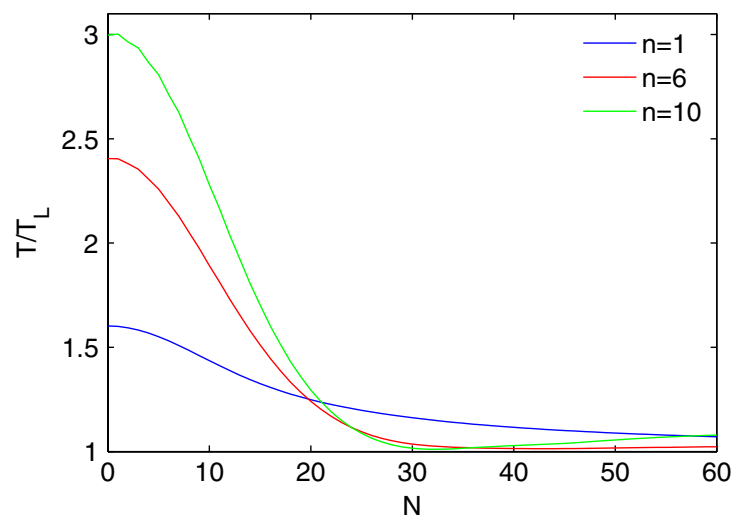

FIG. 6. Normalized TBPs of the electron bunching as a function of the modulator period number for different harmonics of the seed $(n=1,6$, and 10$)$. 

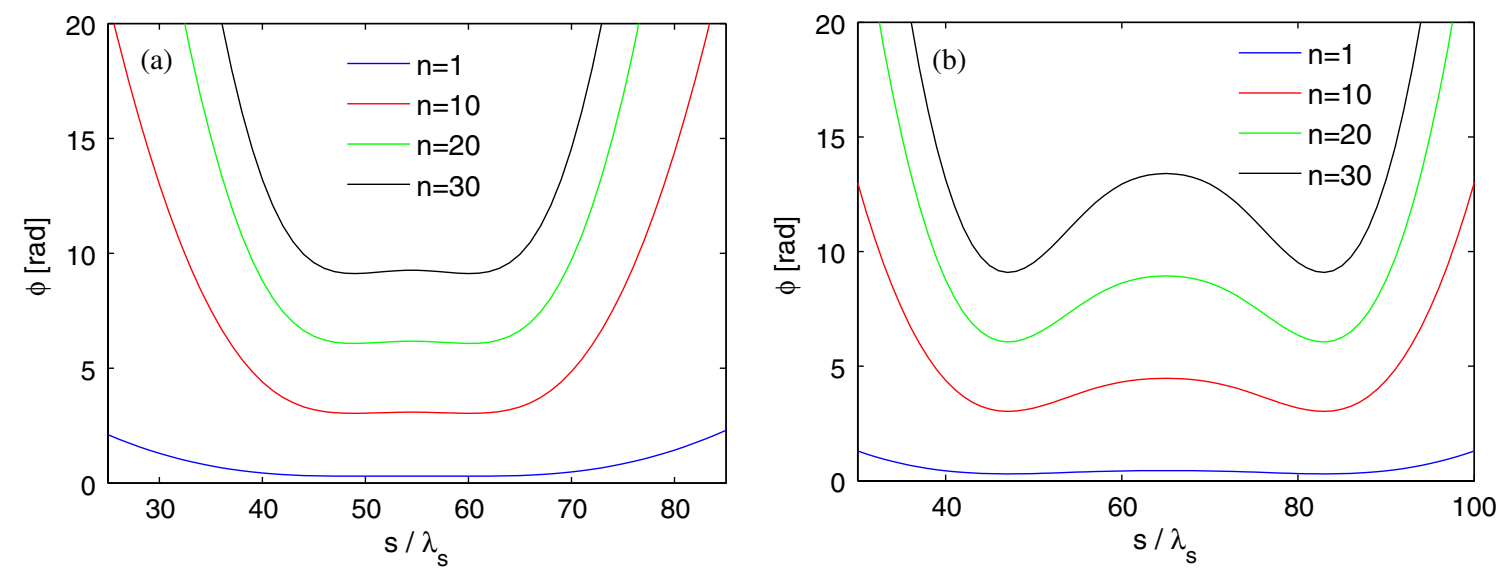

FIG. 7. Phase distributions of the bunching in UR at various harmonics $(n=1,10,20$, and 30$)$ for different period numbers of the modulator: (a) $N=30$; (b) $N=50$.

e.g. $n<10$, but will be amplified by harmonic number and significantly increase the TBPs for high harmonics. So it is necessary to properly set the period number of the modulator to optimize TBP for high harmonic generation. For our case, the optimized period number is around 30 .

Figure 8 gives the spectra of the electron bunching factors at 10th harmonic of the seed. The period number of the modulator is chosen to be $N=30$ to make the TBP reach its minimal value. For convenience of comparison, four cases have been considered: flat phase and quadratic phase with and without slippage effect. It is found that the bandwidth of the spectral bunching for the quadratic phase case is about 3 times broader than that of the flat phase case when the slippage effect is neglected. However, the spectra are nearly the same for the flat and quadratic phase cases when the slippage effect is considered, which implies that the frequency chirp in an ultrashort seed pulse may not

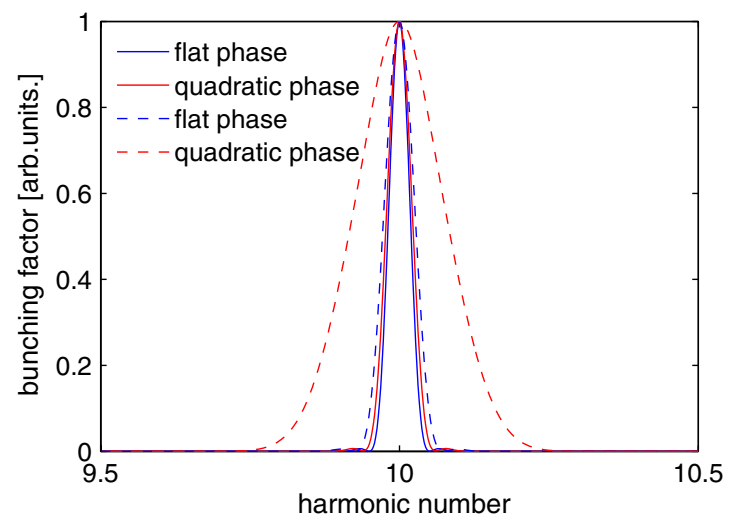

FIG. 8. Bunching factor spectra for different cases: seed laser with flat phase and considering the slippage effect (blue solid line); seed laser with quadratic phase and considering the slippage effect (red solid line); seed laser with flat phase and ignoring the slippage effect (blue dashed line); seed laser with quadratic phase and ignoring the slippage effect (red dashed line). significantly impact the electron bunching for HGHG with a properly chosen period number of the modulator.

The EEHG seeding mechanism shares many similarities with HGHG. In the standard EEHG scheme, the first laserchicane combination filaments the electron beam in phase space. The second laser-chicane stage then simultaneously bunches each filament, resulting in multiple density spikes within each seed wavelength. Here we consider a scenario where the pulse length of the first seed laser is much longer than the second one. There are two benefits of this setup compared to using two ultrashort seed pulses: first, the long seeding pulse in the first modulator will provide a relativity uniform modulation and phase space distribution along the electron beam, which is beneficial to the increase of bunching factor; second, this setup loosens the constraints on the timing control of these two seeding pulses.

The second stage of EEHG is similar to the HGHG process, with the energy separation of the filaments determining the final harmonic number. The frequency chirp in the seed laser will affect the two stages differently [16]: For the first stage, the chirp mainly distorts the separation of the filaments, which decrease the peak value of the bunching factor. However, because the second seed laser still phase locks each set of density spikes, the chirp from the first laser has little effect on the bandwidth of the bunching. Here we focus on the spectral phase of the bunching factor, so a flattop laser pulse in the first stage and a Gaussian pulse with rms length $\sigma_{s}$ in the second stage is assumed. Following the notation of Ref. [6] and assuming the central frequencies of the two seed lasers equal, the final electron beam distribution function after the second DS can be written as

$$
\begin{aligned}
f_{\mathrm{EEHG}}(p, s)= & \frac{N_{0}}{\sqrt{2 \pi}} \exp \left\{-\left[p-A_{2}\left(k_{s} s-B_{2} p\right)\right.\right. \\
& -A_{1} \sin \left[\left(k_{s} s-B_{1} p-B_{2} p\right)\right. \\
& \left.\left.+B_{1} A_{2}\left(k_{s} s-B_{2} p\right)\right]^{2} / 2\right\},
\end{aligned}
$$




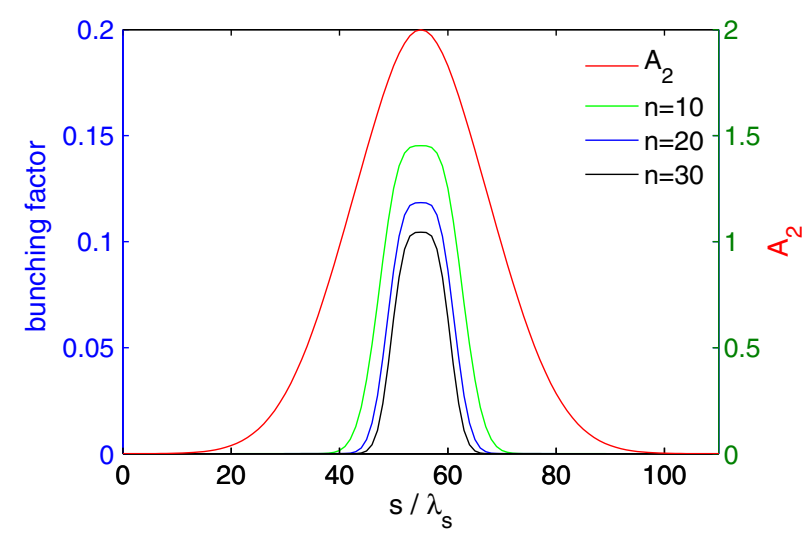

FIG. 9. Local bunching factor distributions along the electron beam for different harmonics of the seed $(n=10,20$, and 30).

where $A_{2}(s)=\gamma(s) / \sigma_{\gamma}$, and the local bunching factor distribution for EEHG is

$$
\begin{aligned}
b(n, s)= & \frac{1}{\sqrt{2 \pi}} \int_{s-l / 2}^{s+l / 2} d s \int_{-\infty}^{\infty} \exp \left(-i n k_{s} s\right) \\
& \times \exp \left\{-\left[p-A_{2}\left(k_{s} s-B_{2} p\right)\right.\right. \\
& -A_{1} \sin \left(k_{s} s-B_{1} p-B_{2} p\right) \\
& \left.\left.+B_{1} A_{2}\left(k_{s} s-B_{2} p\right)\right]^{2} / 2\right\} d p / l .
\end{aligned}
$$

We assume that the energy modulation amplitudes for the two stages are $A_{1}=3, A_{2 m}=2$, where $A_{2 m}$ is the maximal value of $A_{2}$, and the length of each slice is equal to the seed wavelength, $l=\lambda_{s}$. The properties of the second seed pulse are the same as that used in the HGHG case. The optimized strengths of the DSs for $n$th harmonic bunching factor can be calculated using the method given in Ref. [6]. Figure 9 shows the local bunching factor distribution along the electron bunch for different harmonic numbers. As the bunching factor is very sensitive to the energy modulation amplitude at high harmonics, the bunching distributions

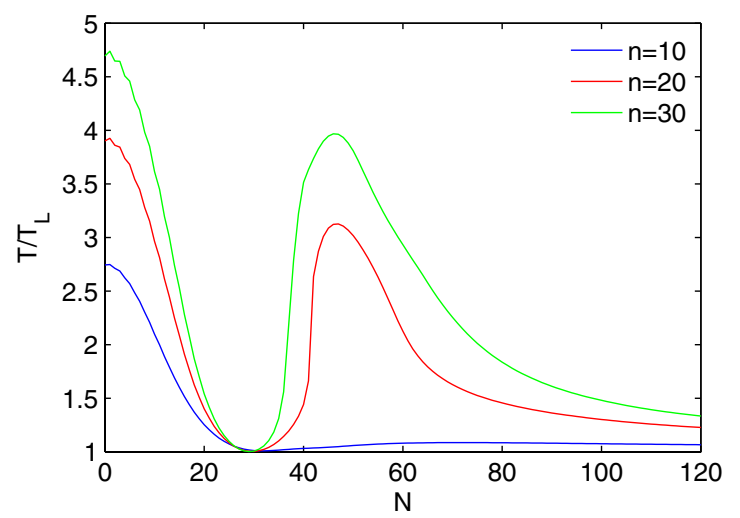

FIG. 10. Normalized TBPs of the electron bunching as a function of the modulator period number for different harmonics of the seed ( $n=10,20$, and 30). have sharp edges at lateral parts of the energy modulation. Figure 10 gives the normalized TBPs of the bunching pulse as a function of the modulator period number for different harmonics. It is found that the optimized period numbers for high harmonics are all around 30. For $n=20$ and 30, the TPBs have peaks around $N=50$ due to the reversed frequency chirp in the UR of energy modulation. After that the TPBs start to decrease again as the period number increase.

\section{3D SIMULATIONS FOR SXFEL}

To verify the theoretical results in previous sections, we have carried out 3D simulations using GENESIS [27] based on the nominal parameters of Shanghai Soft X-ray FEL (SXFEL) project [28]. The SXFEL test facility aims at generating $8.8 \mathrm{~nm}$ FEL from a $264 \mathrm{~nm}$ conventional seed laser through a two-stage cascaded HGHG or a single stage EEHG configuration. The electron beam energy is $840 \mathrm{MeV}$ with emittance of $1 \mathrm{~mm}$ mrad and slice energy spread of about $84 \mathrm{keV}$. The beam peak current is $600 \mathrm{~A}$.

For cascaded HGHG operation, the test facility converts the seed laser at wavelength $\lambda_{\text {seed }}=264 \mathrm{~nm}$ to the FEL at $44 \mathrm{~nm}$ with the first stage HGHG, and it is followed by the second HGHG stage to produce the $8.8 \mathrm{~nm}$ soft $\mathrm{x}$-ray radiation. Here we only consider the first stage HGHG with harmonic up-conversion number of 6 . We assume the pulse length of the seed laser is $16 \mathrm{fs}$ (FWHM), and the period number of the modulator is 30 . For comparison purposes, seed lasers with flat and quadratic spectral phase (linear chirp) distributions have been considered. For the quadratic phase case, we set $\alpha=0.006 / \lambda_{s}{ }^{2}$, which makes TBP of the seed pulse 1.6 times larger than that of a transform-limited pulse with the same pulse length. According to Refs. $[15,16]$, the TBPs of the output radiations should be 2.4 and 4.7 times larger than that of

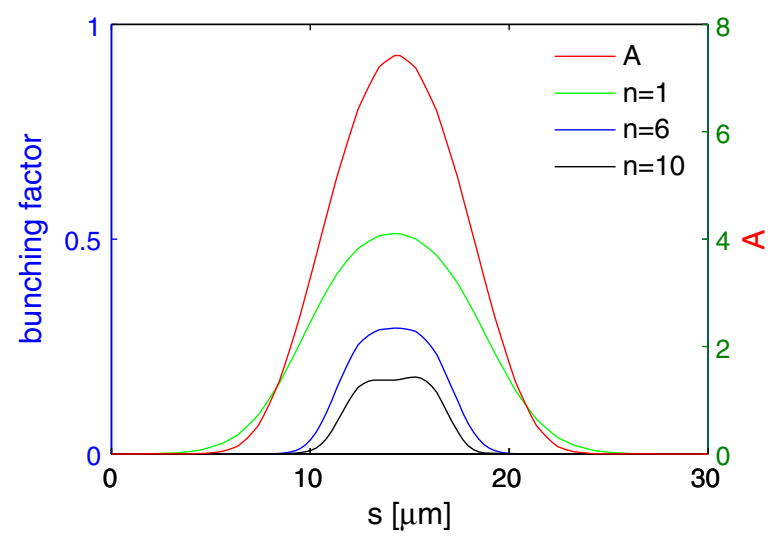

FIG. 11. Simulation results of the energy modulation amplitude and corresponding local bunching factor distributions along the electron beam for different harmonics of the seed $(n=1,6$, and 10). 

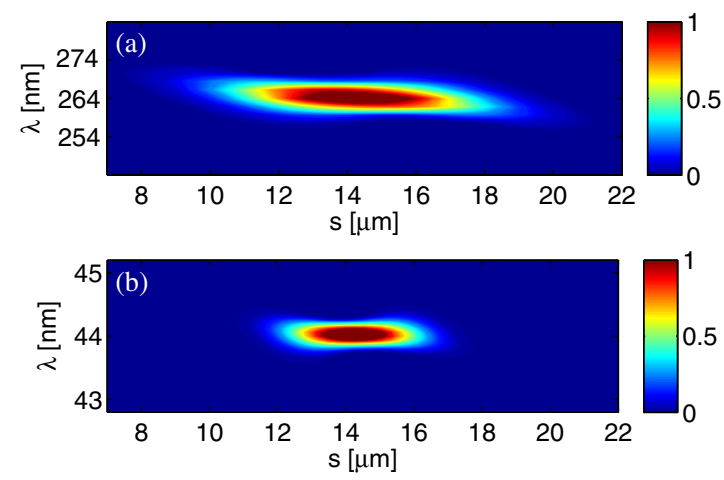

FIG. 12. Wigner distributions of coherent harmonic radiation at fundamental (a) and 6th harmonic (b) of the seed. The FWHM pulse length of the seed laser is $16 \mathrm{fs}$. The frequency chirp in the seed laser pulse is $\alpha=0.006 / \lambda_{s}{ }^{2}$.

transform-limited pulses for 6th and 30th harmonics when ignoring the slippage effect.

After passing through the DS, the electron beam is well bunched. Figure 11 shows the energy modulation amplitude and various bunching factor distributions

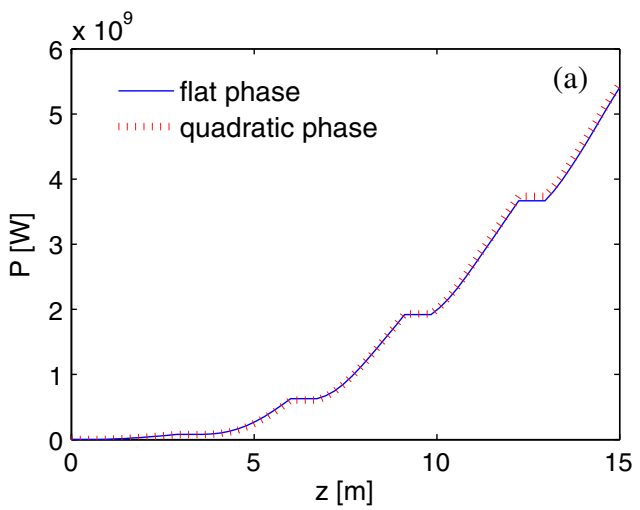

along the electron bunch. One can find that these simulation curves fit quite well with the theoretical calculation results given in Fig. 4. The resonant wavelength of the radiator in the first stage of SXFEL is flexible, so we can make the radiator resonant at the fundamental and 6 th harmonic of the seed. The WDs of the fundamental $(264 \mathrm{~nm})$ and 6 th harmonic $(44 \mathrm{~nm})$ radiations at the very beginning of the radiator for the quadratic phase case are shown in Fig. 12. As the FEL works in the CHG regime $[3,4]$, the radiation power is proportional to the square of the bunching factor. One can clearly see that the frequency chirps occur in the lateral parts of the radiation pulses, which agrees with the theoretical predictions as shown in Fig. 5. As the central part of the radiation pulse with large power has flat phase distribution, the spectrum of the radiation for the quadratic phase case will have little difference from the flat phase case. Figure 13 shows the FEL performance of the 6th harmonic radiation. It is found that the gain curves and spectra for these two cases are nearly the same. The bandwidths of the radiation pulses at saturation are both around $0.2 \%$, which indicates that the initial frequency

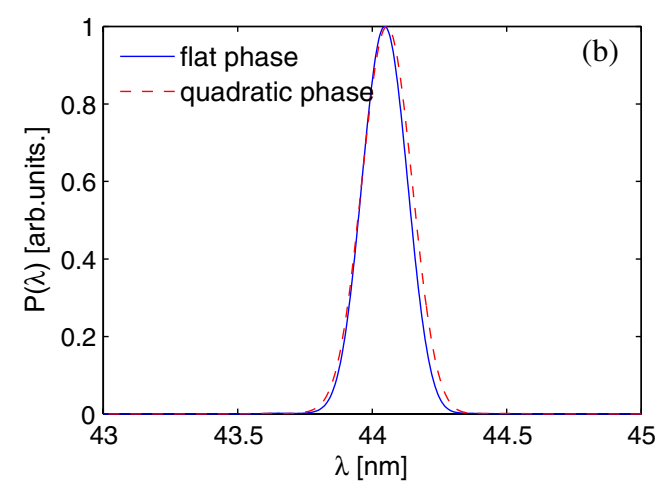

FIG. 13. 6th harmonic radiation performances of HGHG for the 16 fs seed laser pulses with flat phase (blue line) and quadratic phase (red dashed line): (a) FEL gain curves; (b) spectra at saturation.
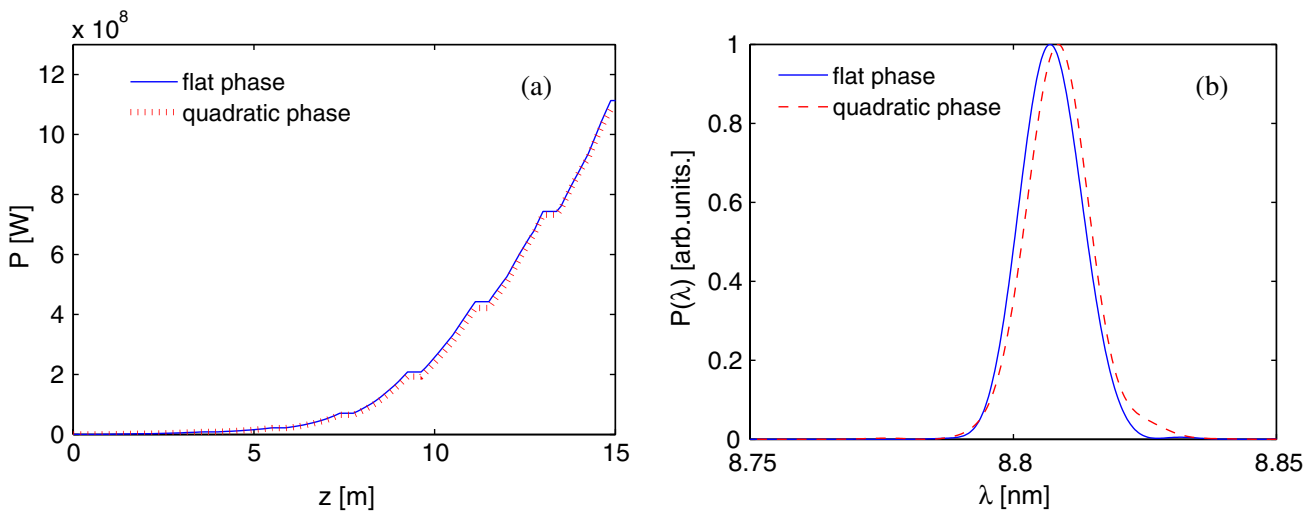

FIG. 14. 30th harmonic radiation performances of EEHG for the $16 \mathrm{fs}$ seed laser pulses with flat phase (blue line) and quadratic phase (red dashed line): (a) FEL gain curves; (b) spectra at saturation. 
chirp in the seed laser does not lead to broadening of the bandwidth of the harmonic radiation of HGHG.

For EEHG operation, we assume that the first seed laser pulse is longer than the electron bunch. The properties of the second seed laser are chosen to be the same as that used in HGHG simulations. The period numbers of the two modulators are both 30 . The energy modulation amplitudes and dispersion strengths in our simulation are set to be $A_{1}=3.5, A_{2}=4, B_{1}=8.86$, $B_{2}=0.28$ to maximize the bunching factor at 30th harmonic of the seed. The corresponding seed laser peak powers are 40 and $80 \mathrm{MW}$, respectively. The performance of the radiation also has a very weak dependence on the chirp in the seed laser, as shown in Fig. 14. The bandwidths of the radiation pulses at saturation are also around $0.2 \%$.

\section{SLIPPAGE BOOSTED BY A SUBHARMONIC MODULATOR}

In the previous section, we have shown that nearly transform-limited high harmonic radiation pulses can be generated via seeded FELs, when the slippage length in the modulator is comparable to the pulse length of the seed laser. For a $16 \mathrm{fs}$ seed laser pulse at $264 \mathrm{~nm}$, the optimized period number is about 30 , which is a reasonable value for modulator design. However, when using a longer seed laser pulse for the generation of narrower bandwidth radiation, this period number is not large enough to compensate the initial frequency chirp induced by the seed. Figure 15 shows the simulated spectra of the 30th harmonic radiations of EEHG with 80 fs (FWHM) seed laser pulses. The frequency chirp in the seed laser pulse is $\alpha=0.0006 / \lambda_{s}{ }^{2}$ for the quadratic phase case, which makes TBP of the seed pulse 3 times larger than that of a transform-limited pulse with the same pulse length. The period number of the modulator is set to be 22 here. Other parameters are the same as that used in the previous section. It is found from Fig. 15 that the

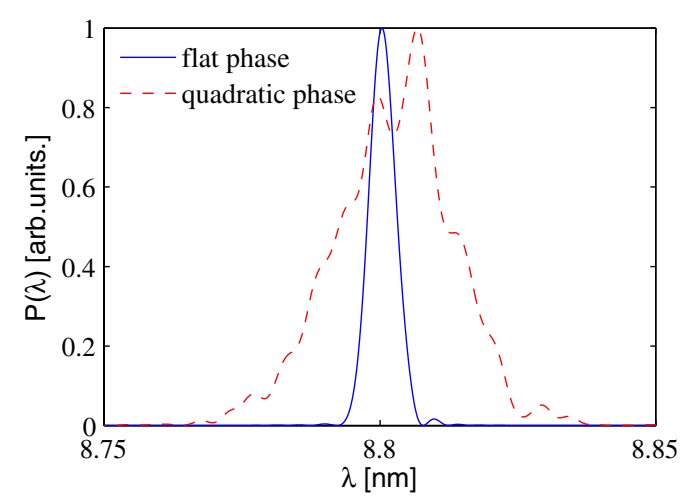

FIG. 15. Spectra of 30th harmonic radiation pulses of EEHG for the $80 \mathrm{fs}$ seed laser pulses with flat phase (blue solid line) and quadratic phase (red dashed line).
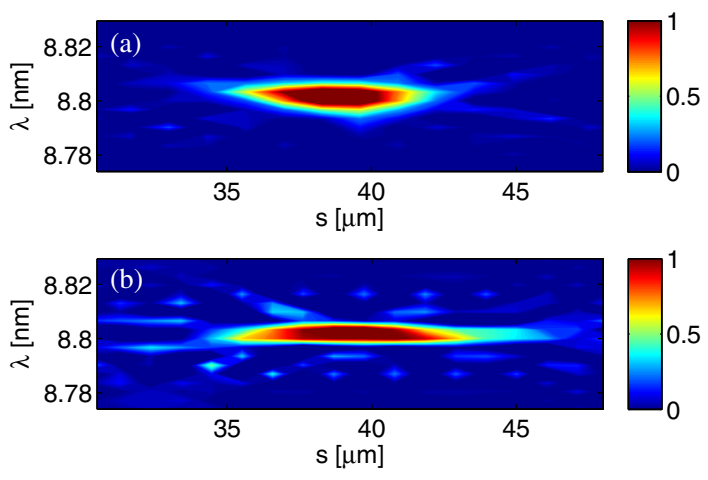

FIG. 16. Wigner distributions of 30th harmonic radiation pulses at the very beginning of the radiator of EEHG for the 80 fs seed laser pulses with flat phase (a) and quadratic phase (b).

bandwidth of the 30th harmonic radiation for the quadratic phase case is about 4.5 times broader than that of the flat phase case. Although the TBP of the radiation pulse has already been reduced by the modulator with 22 periods, the period number still needs to be further increased by about 5 times to fully compensate the frequency chirp induced by the seed laser. However, when the period number is too large, e.g., larger than 50 for SXFEL, the modulator will no longer work in the small-gain regime. In this case, the FEL interaction tends to wash out the fine structures in energy space and will lead to a significant degradation to the quality of the electron bunch.

It has been proposed that undulator sections resonant at subharmonics of the FEL radiation can be used to increase the FEL slippage length, which will lead to a reduced bandwidth of a SASE FEL [29]. Here, we apply the similar idea to the modulator of seeded FEL schemes for the slippage-boosting purpose. Instead of resonant at the fundamental of the seed, the modulator is tuned to an odd subharmonic: $\lambda_{m}=m \lambda_{s}, m=3,5,7, \ldots$, which will increase the slippage length by $m$ times in the modulator while simultaneously keeping the FEL interaction in the small-gain regime.

Here we make the second modulator of EEHG resonant at $1320 \mathrm{~nm}$, which is 5 times longer than the seed wavelength. The peak power of the second seed laser is increased to $180 \mathrm{MW}$ to generate the same energy modulation amplitude $\left(A_{2}=4\right)$ as we have used in the last section. Figure 16 shows Wigner distributions of 30th harmonic radiation pulses at the very beginning of the radiator for flat and quadratic phase cases. One can find that there are no chirps in the radiation for both these two cases, which implies that the initial chirp in the seed laser has been compensated by the subharmonic modulator. Figure 17 gives the FEL performance for these two cases. The output peak powers are at the same level. The bandwidths of the radiation pulses at saturation are both around $0.05 \%$, which is about 4 times narrower than the output 

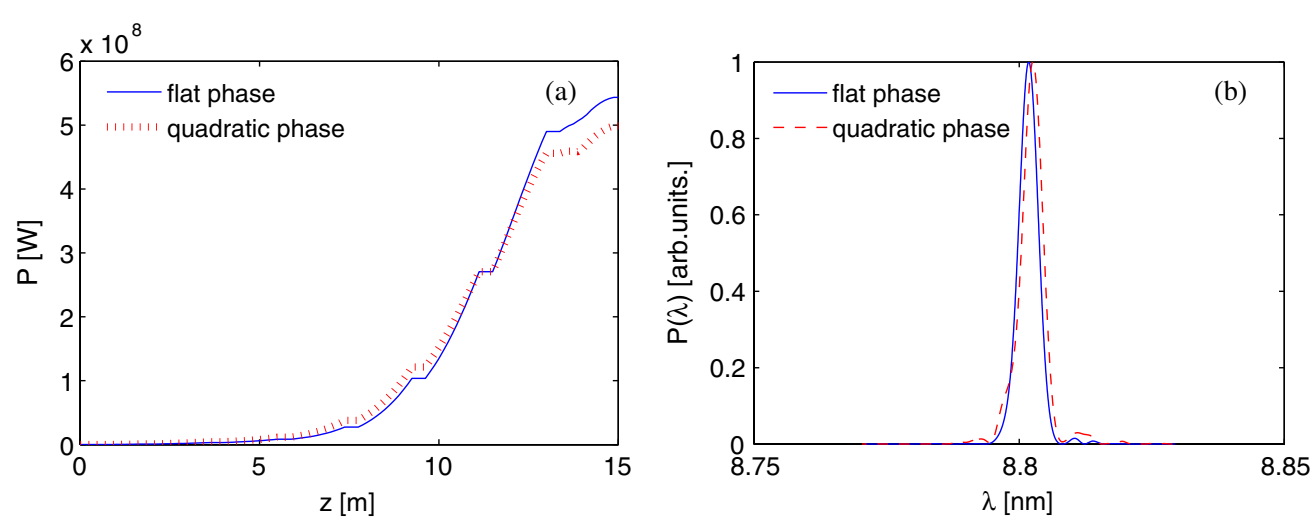

FIG. 17. 30th harmonic radiation performances of EEHG with subharmonic modulator resonant at $1320 \mathrm{~nm}$ for the $80 \mathrm{fs}$ seed laser pulses with flat phase (blue line) and quadratic phase (red dashed line): (a) FEL gain curves; (b) spectra at saturation.

bandwidth of the normal EEHG with a 16 fs seed laser pulse [Fig. 14(b)].

\section{CONCLUSION}

In summary, energy modulations for seeded FEL schemes with frequency chirped seed laser pulses have been studied analytically and numerically. It is found that, by adopting a seed laser with pulse length comparable to the slippage length in the modulator, the initial frequency chirp induced by the imperfect seed laser pulse can be significantly smoothed and the production of nearly transform-limited radiation pulses is possible. 3D simulations have been carried out and the results confirm the theoretical predictions. In this paper, only the seed laser pulse with linear frequency chirp case has been studied. We expect that the slippage effect can also be used to compensate other temporal and spatial errors induced by the seed laser with nonideal properties, e.g., arbitrary spectral phase errors and laser field errors, etc. It should be pointed out that all the conclusions drawn from this study are under the condition of using an ideal beam with constant energy and current. For a realistic electron beam, the nonhomogeneity of current and energy distributions along the electron beam at the exit of the linac is unavoidable. The beam energy chirp will affect HGHG and EEHG differently [30]: nonlinear energy chirp in the electron beam will broaden the bandwidth of a HGHG-FEL significantly, while the EEHG-FEL with optimized parameters can be nearly immune to the energy chirp at high harmonics. The nonuniform distribution of beam current will result in a nonuniform distribution of the output radiation profile, which will also degrade the coherence of a seeded FEL. However, for an ultrashort seed laser with pulse length much shorter than the electron bunch, only a small part of the electron beam is used for lasing. With a sufficiently flat phase space and current distributions in this small part, we can still ignore the effects of the imperfections of the electron bunch on the properties of the output radiation pulse. Further investigations on these issues will be performed in the future.

\section{ACKNOWLEDGMENTS}

The authors would like to thank J. Chen, T. Zhang, L. Shen, and Z. Huang for helpful discussions and comments. This work is supported by Major State Basic Research Development Program of China (973 Program) (Grant No. 2011CB808300) and National Natural Science Foundation of China (Grant No. 10935011). The work of D. Xiang is supported by U.S. DOE Contract No. DEAC02-76SF00515.

[1] P. Emma et al., Nat. Photonics 4, 641 (2010).

[2] T. Ishikawa et al., Nat. Photonics 6, 540 (2012).

[3] B. Girard, Y. Lapierre, J. M. Ortega, C. Bazin, M. Billardon, P. Elleaume, M. Bergher, M. Velghe, and Y. Petroff, Phys. Rev. Lett. 53, 2405 (1984).

[4] L. H. Yu, Phys. Rev. A 44, 5178 (1991).

[5] G. Stupakov, Phys. Rev. Lett. 102, 074801 (2009).

[6] D. Xiang and G. Stupakov, Phys. Rev. ST Accel. Beams 12, 030702 (2009).

[7] M. Labat, M. Hosaka, A. Mochihashi, M. Shimada, M. Katoh, G. Lambert, T. Hara, Y. Takashima, and M.E. Couprie, Eur. Phys. J. D 44, 187 (2007).

[8] E. Allaria, F. Curbis, M. Coreno, M. Danailov, B. Diviacco, C. Spezzani, M. Trovó, and G. De Ninno, Phys. Rev. Lett. 100, 174801 (2008).

[9] N. Čutić, F. Lindau, S. Thorin, and S. Werin, Phys. Rev. ST Accel. Beams 14, 030706 (2011).

[10] L. H. Yu et al., Science 289, 932 (2000).

[11] D. Xiang et al., Phys. Rev. Lett. 105, 114801 (2010).

[12] D. Xiang et al., Phys. Rev. Lett. 108, 024802 (2012).

[13] Z. T. Zhao et al., Nat. Photonics 6, 360 (2012).

[14] E. L. Saldin, E. A. Schneidmiller, and M. V. Yurkov, Opt. Commun. 202, 169 (2002).

[15] G. Geloni, V. Kocharyan, and E. Saldin, arXiv: $1111.1615 \mathrm{v} 1$.

[16] D. Ratner, A. Fry, G. Stupakov, and W. White, Phys. Rev. ST Accel. Beams 15, 030702 (2012).

[17] P. Maine, D. Strickland, P. Bado, M. Pessot, and G. Mourou, IEEE J. Quantum Electron. 24, 398 (1988). 
[18] E. Allaria et al., Nat. Photonics 6, 699 (2012).

[19] C. Feng, M. Zhang, G. Lin, Q. Gu, H. Deng, J. Chen, D. Wang, and Z.T. Zhao, Chin. Sci. Bull. 57, 3423 (2012).

[20] H. Deng, X. Wang, and Z. Dai, Phys. Rev. ST Accel. Beams 11, 040703 (2008).

[21] H. Deng and Z. Dai, J. Phys. D 41, 115503 (2008).

[22] G. De Ninno, B. Mahieu, E. Allaria, L. Giannessi, and S. Spampinati, Phys. Rev. Lett. 110, 064801 (2013).

[23] E. Wigner, Phys. Rev. 40, 749 (1932).

[24] M. J. Bastiaans, Optik (Jena) 82, 173 (1989).

[25] L. H. Yu and J. Wu, Nucl. Instrum. Methods Phys. Res., Sect. A 483, 493 (2002).
[26] C. Feng, T. Zhang, J. Chen, H. Deng, M. Zhang, X. Wang, B. Liu, T. Lan, D. Wang, and Z. T. Zhao, Phys. Rev. ST Accel. Beams 14, 090701 (2011).

[27] S. Reiche, Nucl. Instrum. Methods Phys. Res., Sect. A 429, 243 (1999).

[28] Z. T. Zhao, S. Chen, L. H. Yu, C. Tang, L. Yin, D. Wang, and $\mathrm{Q} . \mathrm{Gu}$, in Proceedings of the 2nd International Particle Accelerator Conference, San Sebastián, Spain (EPS-AG, Spain, 2011), JACOW, p. 3011.

[29] D. Xiang, Y. Ding, Z. Huang, and H. Deng, Phys. Rev. ST Accel. Beams 16, 010703 (2013).

[30] C. Feng, D. Wang, and Z. T. Zhao, in Proceedings of the 3rd International Particle Accelerator Conference, New Orleans, Louisiana, USA, 2012 (IEEE, Piscataway, NJ, 2012), JACOW, p. 1724. 